\section{Photonic Crystal Fabrication}

\section{Photonic Crystals}

Since the 1980s, it has been realized that light can be controlled in an intricate way by nanostructures, hence the term "nanophotonics." For instance, two groups demonstrated that multiple scattering of light can lead to intricate interference phenomena. Of particular interest are three-dimensional photonic crystals. In the ultimate three-dimensional photonic crystal with a band gap, certain frequencies of light are completely forbidden from existence, which makes them the supreme devices to control light. Originally, it was predicted that spontaneous emission of light is forbidden in these nanostructures or that light is localized. Another intriguing aspect of photonic crystals is that by adding an embedded microcavity, photons can be stored or slowed down. Therefore, photonic crystals truly function to "mold the flow of light."

A photonic crystal is a periodic nanostructure made from two different materials that are periodically alternated over length scales on the order of the wavelength of light. Typically, one material is air and the other has a high index of refraction, e.g., a semiconductor. Light with certain wavelengths and wave vectors cannot propagate in such a structure because of Bragg diffraction. This is the condition where light that is reflected off the lattice planes in the crystal interferes constructively. The Bragg condition was first described for X-rays but is also valid for light. In case of a broadband (white light) reflectivity measurement on a photonic crystal, the Bragg condition appears as a peak in the spectrum, also referred to as stop band. No stop band exists when the difference in the refractive index of the constituent materials of the photonic crystal is zero. The stop band gets broader when the difference in refractive indices increases.

An important parameter of photonic crystals is the photonic strength, $S$, defined as the polarizability per unit cell volume. The photonic strength, $S$, is a measure of the interaction strength of photonic crystals with light. A higher $S$ means that the crystal is more strongly interacting with light. Two frequencies form the edges of the stopgap of the photonic crystal $\Delta \omega_{\text {gap }}$. The center of the stopgap is the central frequency $\omega_{\text {gap }}$. By measuring $\omega_{\text {gap }}$ and $\Delta \omega_{\text {gap }}$, the photonic strength $S$ can be determined experimentally:

$$
S=\frac{\Delta \omega_{\text {gap }}}{\omega_{\text {gap }}}
$$

Whereas a stopgap is defined in one direction only, in a photonic band gap crystal a range of forbidden frequencies exists for all directions in three dimensions. In order for a photonic band gap to appear, stopgaps from different lattice planes in the crystal overlap and couple. For this to occur, the photonic strength $S$ of the crystal, and thus the difference in refractive indices of the two photonic crystal materials, must be high enough. The rule of thumb is that the photonic strength should exceed $S \geq 0.20$ for a photonic band gap to appear. In nanophotonics, one of the targets is to obtain three-dimensional photonic crystals with band gaps that are as broad as possible. Some examples of applications pursued with photonic crystals are the following:

- Thresholdless cavity lasers that are interesting in, e.g., miniaturized devices because of their high efficiency and low noise.

- Optical components to control the propagation of light, especially fibers, polarizing beamsplitters, and waveguides.

- Devices for detection and sensing, including detection of gas molecules, highly sensitive fluorescence detection and the detection of single particles.

- To change the color or the spontaneous emission rate at which light sources embedded inside the photonic crystals emit.

- (Optically) switching three-dimensional photonic crystals allows, for example, dynamic control of the emission of light sources that are inside the photonic crystal, e.g., the emission can be turned on or off.

- Ultimately, photonic crystals are predicted to contribute to all-optical computers where bits are encoded as light. Compared to traditional electronic computers, all-optical systems should be more energy efficient, faster, and have larger bandwidths.

\section{Inverse Opals}

Many different types of three-dimensional photonic crystals have been conceived but of particular interest are those that potentially provide three-dimensional photonic band gaps, as these offer ultimate control of light in all three dimensions simultaneously. Among the first structures considered as promising photonic crystals were face-centered cubic (fcc) crystals of colloidal spheres. These crystals are routinely obtained by different methods of self-assembly. Two types of fcc crystals of spheres are distinguished: (a) colloidal crystals that consist of separate spheres that perform Brownian motion while suspended in a liquid and (b) artificial opals where the spheres are immobilized as they are in close-packed contact and surrounded by air, as shown in Fig. 1.

Artificial opals made from self-assembled colloidal spheres are of considerable scientific and technological interest as photonic crystals, as components of light sources, solar cells, and chemical sensors, as well as in the field of plasmonics. Although colloidal 


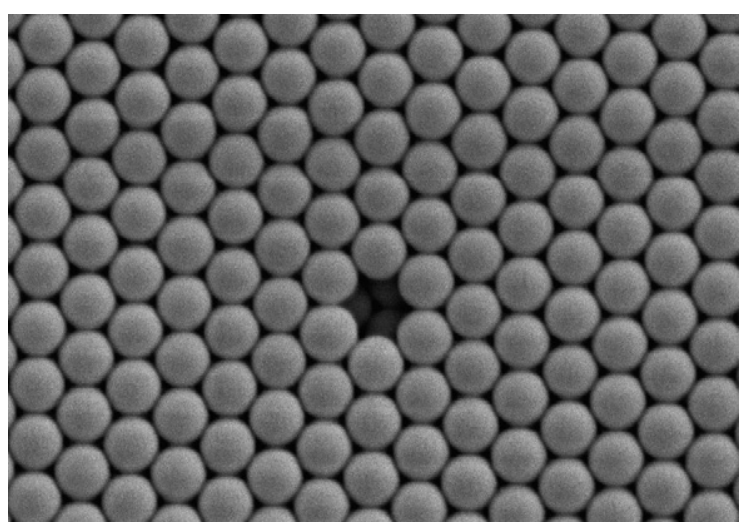

Figure 1

Scanning electron micrograph of the surface of a polystyrene artificial opal. Artificial opals are generally obtained by different methods of self-assembly. The spheres have a radius of $439 \mathrm{~nm}$. A surface cavity consisting of a vacancy is apparent that is observed by chance in a self-organizing opal. A method has been demonstrated to make point defects by design.

crystals and artificial opals are interesting as photonic structures in their own right, they cannot form a photonic band gap due to the limited photonic strength of these crystals; for an artificial opal of polystyrene spheres, the photonic strength is approximately $S=0.06$, whereas for photonic band gap formation, the photonic strength should be higher than 0.2 .

Although it was originally suggested that it is impossible to obtain a band gap in fcc photonic crystals, it was realized in 1992 that in inverse opals it is possible to obtain a photonic band gap. Inverse opals consist of fcc-stacked spheres of air, embedded in a backbone of a higher index of refraction material (see Fig. 2). These inverse artificial opals can be obtained by using fcc colloidal sphere crystals as a template and filling them with a high index of refraction material, followed by removal of the opal template. Examples include inverse opals of metal oxides such as titanium dioxide, carbon, cadmium selenide, and even of metals. Alternative templates for inversion can be obtained by, e.g., holographic lithography.

In inverse opals, the index of refraction contrast must exceed 2.8 in order to obtain a photonic band gap. A material that meets this requirement is silicon with a refractive index of 3.5. Consequently, inverse opals of silicon are promising candidates to display a photonic band gap. Unfortunately, even for silicon, the calculated maximum width of the band gap in fcc inverse opals remains limited to $12 \%$. The photonic band gap in fcc inverse opals is very sensitive to fabrication-induced disorder, which limits the usefulness of these structures when large band gaps are required.

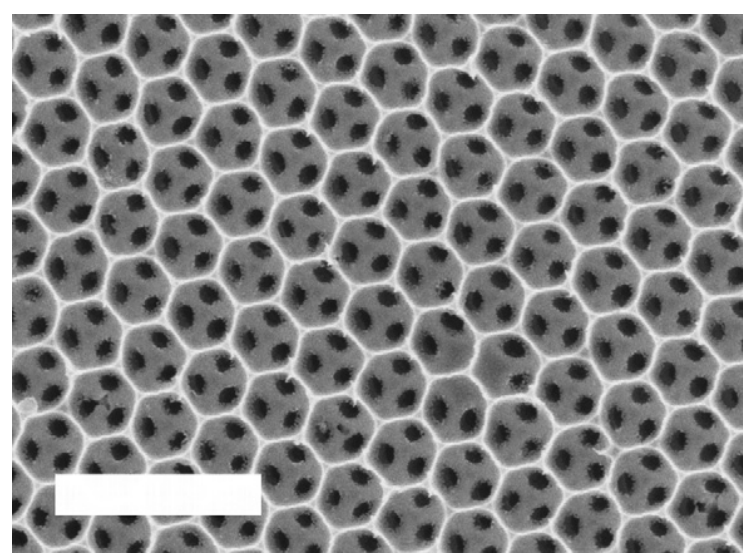

\section{Figure 2}

Scanning electron micrograph of the surface of an inverse opal. Inverse opals are obtained by using fcc colloidal sphere crystals as a template and filling them with a high index of refraction material, in this case titanium dioxide, followed by removal of the template. The scale bar equals $1 \mu \mathrm{m}$. Reproduced with permission from Lodahl $\mathrm{P}$, van Driel A F, Nikolaev I S, Irman A, Overgaag K,

Vanmaekelbergh D, Vos W L 2004 Controlling the dynamics of spontaneous emission from quantum dots by photonic crystals. Nature 430, 654-7. Copyright Wiley.

\section{Simple Cubic Photonic Crystals}

Different varieties of three-dimensional photonic crystals with a simple cubic lattice have been suggested. Among the proposed building blocks are spheres, air spheres, square rods, circular rods, and many other motifs. Examples of simple cubic crystals are beautiful air sphere and square rod crystals. Unfortunately, the maximum width of the band gap of simple cubic photonic crystals remains limited: a maximum width of the (second-order) band gap of $13 \%$ is predicted for an optimized geometry. Moreover, one can expect these second-order band gaps to be sensitive to fabrication-induced disorder, similar to the band gap in fcc air sphere crystals.

\section{Diamond-Like Photonic Crystals}

The so-called diamond-like crystal structure has received a great deal of attention because of its large calculated width of the band gap, and the property that the photonic band gap already opens at an index of refraction contrast of 1.9 compared to 2.8 for fcc air sphere crystals. The use of diamond lattices in photonic crystals was introduced by Ho et al. and was comprehensively reviewed. Some examples of fabricated photonic crystals with a diamond-like structure are the following:

- An early type of diamond-structured photonic crystal is obtained by milling or etching pores in 
separate steps in three directions. In 1995, such crystals were fabricated in GaAs and GaAsP. In a subsequent study, similar crystals were shown to be photonic, but a band gap remains to be demonstrated. For such a crystal made from silicon, the calculated band gaps have a relative width of up to $19 \%$. Later, such a structure was fabricated using a combination of chemical etching and focused ion beam milling. This photonic crystal shows promising reflectivity peaks around $\lambda=3 \mu \mathrm{m}$.

- Diamond-like woodpile structures were introduced by Ho et al. in 1994. They are fabricated by stacking layers of dielectric rods on top of each other in sequential steps (see Fig. 3). The fabricated structures consist of stacked and rectangular touching rods. The maximum band gap width is limited to approximately $18 \%$ for structures in silicon.

- Two groups have described "spiral diamond" crystals, consisting of circular spirals. One of the techniques proposed to fabricate the structures is glancing angle deposition. Subsequent inversion of the square spirals with silicon yields a crystal with a calculated relative width of the band gap of approximately $24 \%$.

- It has been attempted to fabricate diamond-like photonic crystals with dielectric spheres. For example, nanorobotic manipulation was used to obtain a pretty but small five-layer crystal.

- Three-dimensional woodpile crystals were fabricated by a double-angled etching technique. By two consecutive cryogenic reactive ion etch steps of $45^{\circ}$

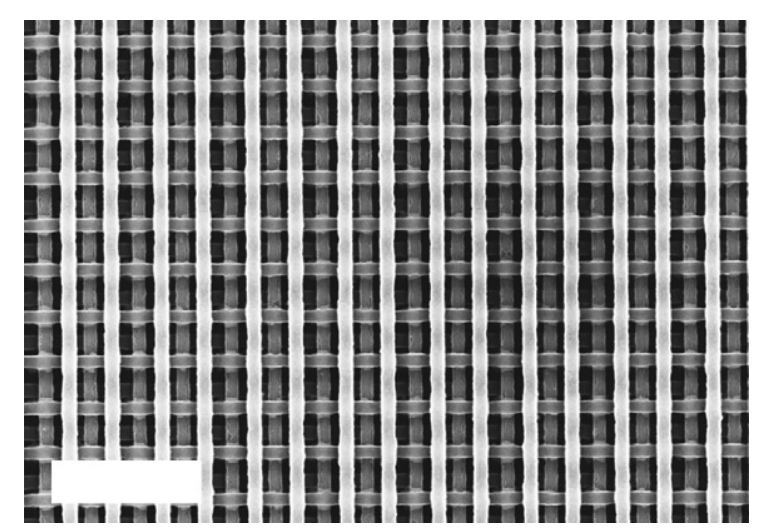

\section{Figure 3.}

Scanning electron micrograph of the surface of a woodpile photonic crystal fabricated by Fleming and Lin (1999). These crystals are obtained by stacking layers of dielectric rods on top of each other in sequential steps using conventional semiconductor processes. The scale bar equals $2 \mu \mathrm{m}$. Reproduced with permission from Harding P J, Euser T G, Vos W L 2009 Identification of competing ultrafast all-optical switching mechanisms in Si woodpile photonic crystals. J. Opt. Soc. Am. B 26, 610-19. Copyright OSR. with respect to the wafer surface, at an angle of $90^{\circ}$ between the two etch directions, a thin photonic crystal was obtained.

\section{Inverse Woodpile Photonic Crystals}

The most promising type of diamond-like photonic crystals was introduced by Ho et al. These structures are referred to as inverse woodpile photonic crystals and consist of two geometrically identical arrays of pores perpendicular to each other in a high refractive index material (see Fig. 4). They differ from woodpile crystals in the sense that the filling fraction of high refractive index material is optimized and that the pores may overlap, which is required to obtain a maximum band gap. Inverse woodpile photonic crystals are very interesting because of their conceptual ease of fabrication by only etching in two directions and their high photonic strength, resulting in a broad band gap with a relative width of more than $25 \%$.

A method was proposed to fabricate such inverse woodpile structures by using macroporous silicon and subsequently focused ion beam milling. Later, by using the proposed method, a crystal was obtained, which was one of the first fabricated inverse woodpiles. It was pointed out that the structure was misaligned, which results in a reduced calculated width of the band gap. A reflectivity spectrum was shown with a $>40 \%$ reflectivity peak. This peak corresponds to a calculated stopgap in the direction that was measured. This result shows that these crystals are reasonably strongly photonic. Inverse woodpiles can also be obtained by solely using focused ion beam milling.

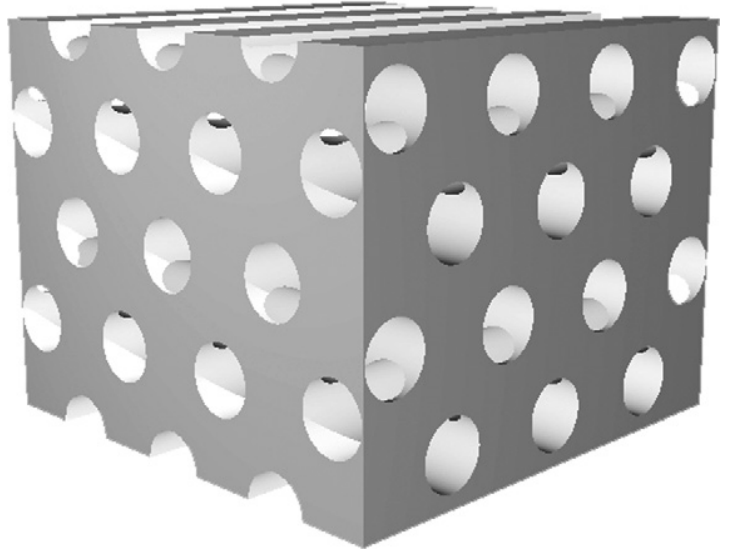

Figure 4

Three-dimensional representation of a cubic inverse woodpile photonic crystal. These structures consist of two geometrically identical arrays of pores perpendicular to each other in a high refractive index material. 
Recently, an alternative method was described to fabricate similar crystals with pores that have an elliptical cross section. This method involves direct laser writing and silicon dioxide-assisted deposition of polycrystalline silicon. Optical transmittance and reflectivity experiments show that this structure is indeed strongly photonic. However, the optical experiments were performed along only one direction and hence do not confirm whether a photonic band gap exists in this structure. With further calculations, it is predicted that the structure can be improved to obtain relative widths of the band gap of $18 \%$.

These examples show that several inverse woodpile crystals have successfully been fabricated. However, in none of these cases was a photonic band gap convincingly demonstrated, which remains an inspiring goal for the (near) future.

\section{Optical Cavities in a Three-Dimensional Photonic Crystal}

One of the great promises of nanophotonics is the possibility that light is trapped in optical cavities inside three-dimensional photonic band gap crystals. An optical cavity in a photonic crystal can be manufactured by either adding or removing high refractive index material, which yields a donor or an acceptor, respectively. The mirrors of the optical cavities are formed by the surrounding photonic crystal with a band gap. It has been predicted that light is trapped in such a photonic crystal cavity within a tiny volume as small as $1 / 100^{\text {th }}$ of a wavelength cubed. It is a challenge to embed such controlled point defects inside three-dimensional photonic band gap crystals to achieve functional systems.

The first experimental observations of donor and acceptor modes in fcc crystals were reported for microwave frequencies. Examples of fabricated defects on a micrometer scale include the incorporation of point defects in woodpile structures. Also, a method was proposed to obtain point defects in artificial opals using focused ion beam milling. Thus, photonic band gap crystals are on a fast lane toward a bright future.

See also: Photonic Crystals: Principles and Applications; Photonic-lattice Filament

\section{Bibliography}

Blanco A, Chomski E, Grabtchak S, Ibisate M, John S, Leonard S W, Lopéz C, Meseguer F, Míguez H, Mondia J P, Ozin G A, Toader O, van Driel H M 2000 Large-scale synthesis of a silicon photonic crystal with a complete three-dimensional bandgap near 1.5 micrometres. Nature 405, 437-40

Bragg W L 1913 Diffraction of short electromagnetic waves by a crystal. Proc. Camb. Phil. Soc. 17, 43-57
Braun P V, Wiltzius P 1999 Electrochemically grown photonic crystals. Nature 402, 603-4

Campbell M, Sharp D N, Harrison M T, Denning R G, Turberfield A J 2000 Fabrication of photonic crystals for the visible spectrum by holographic lithography. Nature 404, 53-6

Chelnokov A, Wang K, Rowson S, Garoche P, Lourtioz J M 2000 Near-infrared Yablonovite-like photonic crystals by focused-ion-beam etching of macroporous silicon. Appl. Phys. Lett. 77 (19), 2943-5

Cheng C C, Scherer A 1995 Fabrication of photonic bandgap crystals. J. Vac. Sci. Technol. B 13(6), 2696-700

Chutinan A, Noda S 1998 Spiral three-dimensional photonic band-gap structure. Phys. Rev. B 57 (4), R2006-8

Fleming J G, Lin S Y 1999 Three-dimensional photonic crystal with a stop band from 1.35 to $1.95 \mu \mathrm{m}$. Optics Lett. 24 (1), 49-51

García-Santamaría F, Miyazaki H T, Urquía A, Ibisate M, Belmonte M, Shinya N, Meseguer F, López C 2002 Nanorobotic manipulation of microspheres for on-chip diamond architectures. Adv. Mater. 14(16), 1144-7

Harding P J, Euser T G, Vos W L 2009 Identification of competing ultrafast all-optical switching mechanisms in $\mathrm{Si}$ woodpile photonic crystals. J. Opt. Soc. Am. B 26, 610-19

Hermatschweiler M, Ledermann A, Ozin G A, Wegener M, von Freymann G 2007 Fabrication of silicon inverse woodpile photonic crystals. Adv. Funct. Mater. 17, 2273-7

Hillebrand R, Senz S, Hergert W, Gösele U 2003 Macroporous silicon-based three-dimensional photonic crystal with a large complete band gap. J. Appl. Phys. 94(4), 2758-60

Ho K M, Chan C T, Soukoulis C M 1990 Existence of a photonic gap in periodic dielectric structures. Phys. Rev. Lett. $\mathbf{6 5}(25), 3152-5$

Ho K M, Chan C T, Soukoulis C M, Biswas R, Sigalas M 1994 Photonic band gaps in three dimensions: new layer-bylayer periodic structures. Solid State Commun. 89(5), 413-16

Holland B T, Blanford C F, Stein A 1998 Synthesis of macroporous minerals with highly ordered three-dimensional arrays of spherical voids. Science 281, 538-40

Jiang P, Bertone J F, Hwang K, Colvin V 1999 Single-crystal colloidal multilayers of controlled thickness. Chem. Mater. $11(8), 2132-40$

Joannopoulos J D, Meade R D, Winn J N 1995 Photonic Crystals - Molding the Flow of Light. Princeton University Press, Princeton, NJ

John S 1987 Strong localization of photons in certain disordered dielectric superlattices. Phys. Rev. Lett. 58, 2486-9

Koenderink A F 2003 Emission and transport of light in photonic crystals. Ph.D. Thesis, University of Amsterdam

Kulinowski K M, Jiang P, Vaswani H, Colvin V L 2000 Porous metals from colloidal templates. Adv. Mater. 12(11), 833-8

Lin S Y, Fleming J G, Lin R, Sigalas M M, Biswas R, Ho K M 2001 Complete three-dimensional photonic bandgap in a simple cubic structure. J. Opt. Soc. Am. B 18(1), $32-5$

Lodahl P, van Driel A F, Nikolaev I S, Irman A, Overgaag K, Vanmaekelbergh D, Vos W L 2004 Controlling the dynamics of spontaneous emission from quantum dots by photonic crystals. Nature 430, 654-7

Maldovan M, Thomas E L 2004 Diamond-structured photonic crystals. Nat. Mater. 3, 593-600

Maldovan M, Thomas E L 2005 Photonic crystals: six connected dielectric networks with simple cubic symmetry. $J$. Opt. Soc. Am. B 22 (2), 466-73 
Matthias S, Müller F, Jamois C, Wehrspohn R B, Gösele U 2004 Large-area three-dimensional structuring by electrochemical etching and lithography. Adv. Mater. 16(23-24), 2166-70

Ogawa S P, Imada M, Yoshimoto S, Okano M, Noda S 2004 Control of light emission by $3 \mathrm{D}$ photonic crystals. Science 305(5681), 227-9

Sanders J V 1964 Colour of precious opal. Nature 204, 1151-3

Schilling J, White J, Scherer A, Stupian G, Hillebrand R, Gösele U 2005 Three-dimensional macroporous silicon photonic crystal with large photonic band gap. Appl. Phys. Lett. 86. 011101-1-011101-3

Sözüer H S, Haus J W, Inguva R 1992 Photonic bands: convergence problems with the plane-wave method. Phys. Rev. B 45(24), 13962-72

Takahashi S, Okano M, Imada M, Noda S 2006 Threedimensional photonic crystals based on double-angled etching and wafer-fusion techniques. Appl. Phys. Lett. 89, 1231061-3

Tjerkstra R W, Segerink F B, Kelly J J, Vos W L 2008 Fabrication of three-dimensional nanostructures by focused ion beam milling. J. Vac. Sci. Technol. B 26(3), 973-7

Toader O, John S 2001 Proposed square spiral microfabrication architecture for large three-dimensional photonic band gap crystals. Science 292, 1133-5

Vahala K J 2003 Optical microcavities. Nature 424, 839-46

van Albada M P, Lagendijk A 1985 Observation of weak localization of light in a random medium. Phys. Rev. Lett. 55(24), 2692-5

Villeneuve P R, Fan S H, Joannopoulos J D 1996 Microcavities in photonic crystals: mode symmetry, tunability, and coupling efficiency. Phys. Rev. B 54(11), 7837-42

Vlasov Y A, Bo X Z, Sturm J C, Norris D J 2001 On-chip natural assembly of silicon photonic band gap crystals. $\mathrm{Na}$ ture 414, 289-93
Vos W L, Megens M, van Kats C M, Bosëcke P 1997 X-ray diffraction of photonic colloidal single crystals. Langmuir 13, 6004-8

Vos W L, Sprik R, van Blaaderen A, Imhof A, Lagendijk A, Wegdam G H 1996 Strong effects of photonic band structures on the diffraction of colloidal crystals. Phys. Rev. B 53, 16231-5

Wijnhoven J E G J, Vos W L 1998 Preparation of photonic crystals made of air spheres in titania. Science 281, 802-4

Woldering L A, Otter A M, Husken B H, Vos W L 2006 Focused ion beam milling of nanocavities in single colloidal particles and self-assembled opals. Nanotechnology 17, 5717-21

Woldering L A, Mosk A P, Tjerkstra R W, Vos W L 2009 The influence of fabrication deviations on the photonic band gap of three-dimensional inverse woodpile nanostructures. J. Appl. Phys. 105, 093108-1-093108-10

Wolf P, Maret G 1985 Weak localization and coherent backscattering of photons in disordered media. Phys. Rev. Lett. 55 (24), 2696-9

Yablonovitch E 1987 Inhibited spontaneous emission in solidstate physics and electronics. Phys. Rev. Lett. 58(20), 2059-62

Yablonovitch E, Gmitter T J, Leung K M 1991a Photonic band structure: the face-centered-cubic case employing nonspherical atoms. Phys. Rev. Lett. 67 (17), 2295-8

Yablonovitch E, Gmitter T J, Meade R D, Rappe A, Brommer K D, Joannopoulos J D 1991b Donor and acceptor modes in photonic band structure. Phys. Rev. Lett. 67 (24), 3380-3

Zakhidov A A, Baughman R H, Iqbal Z, Cui C X, Khayrullin I, Dantas S O, Marti I, Ralchenko V G 1998 Carbon structures with three-dimensional periodicity at optical wavelengths. Science 282 (5390), 897-901

L. A. Woldering, R. W. Tjerkstra, and W. L. Vos

Copyright (C) 2011 Elsevier Ltd.

All rights reserved. No part of this publication may be reproduced, stored in any retrieval system or transmitted in any form or by any means: electronic, electrostatic, magnetic tape, mechanical, photocopying, recording or otherwise, without permission in writing from the publishers.

Encyclopedia of Materials: Science and Technology ISBN: 978-0-08-043152-9

pp. $1-5$ 